Quim. Nova, Vol. 27, No. 3, 456-460, 2004

\title{
BIFOSFONATOS (BFs) COMO TRANSPORTADORES OSTEOTRÓPICOS NO PLANEJAMENTO DE FÁRMACOS DIRIGIDOS
}

Lúcia Fioravanti Castro, Antonio Távora de Albuquerque Silva e Man-Chin Chung*

Departamento de Fármacos e Medicamentos, Faculdade de Ciências Farmacêuticas, Universidade Estadual Paulista "Júlio de Mesquista Filho", CP 502, 14801-902 Araraquara - SP

Antonio Gilberto Ferreira

Departamento de Química, Instituto de Química, Universidade Federal de São Carlos, CP 676, 13565-905 São Carlos - SP

Elizabeth Igne Ferreira

Departamento de Farmácia, Faculdade de Ciências Farmacêuticas, Universidade de São Paulo, CP 66083, 05389-970 São Paulo - SP

Recebido em 14/4/03; aceito em 21/10/03

\begin{abstract}
BISPHOSPHONATES AS OSTEOTROPIC CARRIERS FOR DESIGNING SITE-DIRECTED DRUGS. Drug therapy involving bone tissue diseases is difficult, calling for the design of specific drugs. The present paper is a brief review of a new site-directed system termed ODDS (osteotropic drug delivery system), based on a latenciation process, using bisphosphonates as bone carriers. This is an important tool for the rational prodrug design for obtaining selective drugs.
\end{abstract}

Keywords: bisphosphonates; prodrugs; ODDS.

\section{INTRODUÇÃO}

A liberação seletiva de fármacos no local de ação via pró-fármacos tem gerado interesse considerável para aumentar a potência e diminuir os efeitos adversos dos protótipos dos quais derivam. Embora muitas formas de pró-fármacos seletivos tenham sido desenvolvidas, o tecido ósseo ainda permanecia como um alvo limitado, devido às suas propriedades biológicas e à falta de um sistema circulatório semelhante ao de outros tecidos ${ }^{1}$.

O tecido ósseo adulto é distinguido de outros tecidos pela presença de fosfato e cálcio mineral, como a hidroxiapatita $\left[\mathrm{Ca}_{10}\left(\mathrm{PO}_{4}\right)_{6}(\mathrm{OH})_{2}\right]$, que incorpora em sua estrutura outros íons e sais, sendo o principal componente mineral constituinte do osso e o elemento essencial responsável pela função de apoio mecânico ${ }^{2-4}$.

Devido à dinâmica do tecido ósseo, este é continuamente desgastado pela atividade osteoclástica e substituído pela atividade osteoblástica, ambas reguladas por fatores sistêmicos e locais ${ }^{5-6}$.

Como qualquer outro tecido, os ossos são suscetíveis a doenças que influenciam a atividade osteoclástica, como o hiperparatiroidismo e câncer, sendo a hipercalcemia resultado de destruição direta e localizada do tecido ósseo por células neoplásicas ${ }^{7}$. Exemplos primários são a artrite reumatóide (AR) e a lesão espinhal, nas quais se observa a perda gradual e rápida de massa óssea ${ }^{8,9}$.

Recentemente, propôs-se um novo e promissor sistema de liberação de fármacos para atingir o tecido ósseo via pró-fármacos denominado "Osteotropic Drug Delivery System (ODDS)", que utiliza os bifosfonatos como transportadores ${ }^{10}$.

Os bifosfonatos (BFs) são conhecidos por sua alta afinidade pela hidroxiapatita. Esta propriedade trópica óssea dos BFs possibilitou sua utilização no planejamento de formas latentes de liberação de fármacos nas estruturas ósseas ou na medula óssea ${ }^{1}$.

Os pirofosfatos (P-O-P) são reguladores fisiológicos da calcificação e reabsorção óssea, naturalmente presentes no soro e na urina. A ação dos bifosfonatos deve-se à sua semelhança estrutural com este grupo de compostos $^{11-13}$ (Figura 1).

\footnotetext{
*e-mail: chungmc@fcfar.unesp.br
}

O trabalho nesta área tem-se concentrado na liberação de radioisótopos, mas, atualmente, as pesquisas compreendem a liberação de outras moléculas, cuja atividade farmacológica é bem definida. Estas incluem agentes antineoplásicos, anabolizantes, antiinflamatórios e, recentemente, proteínas ${ }^{4}$.<smiles>[R]C([R])(P(=O)(O)O)P(=O)(O)O</smiles>

Figura 1. Estrutura química dos ácidos bifosfônico e pirofosfórico

A descoberta de que os BFs podiam inibir a reabsorção óssea foi realizada em 1960, após tentativas para identificar agentes que fossem semelhantes ao pirofosfato e que pudessem também regular a calcificação óssea, sendo, assim, potencialmente úteis para a prevenção de calcificação heterotópica ${ }^{12}$.

Os bifosfonatos parecem prevenir a calcificação por um mecanismo físico-químico, agindo como cristais após adsorção na superfície óssea. Atuam, também, inibindo a reabsorção do osso por efeito nas células dos osteoclastos, conforme mostra a Figura $2^{12,14}$.

O pirofosfato e os polifosfatos são incapazes de inibir a calcificação ectópica in vivo, quando administrados oralmente, devido à hidrólise do pirofosfato no trato gastrintestinal. Os BFs são análogos não-hidrolisáveis de pirofosfato, porque contêm ligações estáveis P-C-P no lugar de ligações lábeis P-O-P. Constituem-se, portanto, em compostos com as propriedades antidesmineralizantes do pirofosfato, mas resistentes à hidrólise ${ }^{12}$.

A capacidade dos BFs em se ligar ao osso, prevenindo o crescimento e a dissolução de cristais, é maior quando $\mathrm{R}_{1}=\mathrm{OH}$ (etidronato) ao invés de $\mathrm{Cl}$ (clodronato) ${ }^{12,15,16}$.

Embora a estrutura da cadeia lateral de $\mathrm{R}_{2}$ seja a maior determinante de potência anti-reabsorção, ambos os grupos fosfonatos também são necessários para a atividade dos fármacos. Alterações em um ou outro 

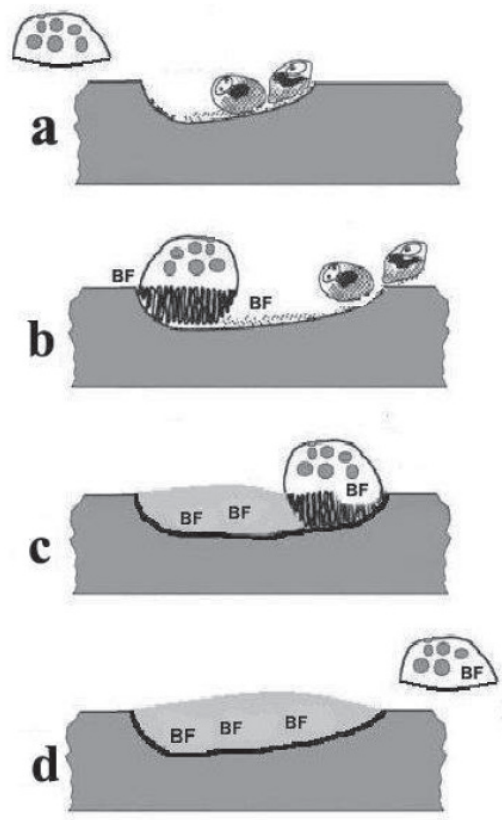

Figura 2. Remodelagem óssea e bifosfonatos (BF): a) ação dos osteoclastos na reabsorção óssea, b) e c) atividade dos osteoblastos na formação óssea utilizando os bifosfonatos e d) final da remodelagem

grupo fosfonato reduzem a afinidade para os ossos. Por exemplo, a substituição de um grupo hidroxila de um dos fosfonatos por um grupo metila formando fosfonofosfinato, notadamente reduz a afinidade e potencia a anti-reabsorção óssea ${ }^{12,17}$. Alterações nos dois grupos fosfonatos, resultando em bifosfinato, conduzem à perda da afinidade óssea e da atividade anti-reabsorção in vivo ${ }^{14,18}$.

Os dois grupos fosfonatos, em conjunto com o grupo hidroxila da cadeia lateral $R_{1}$, conferem alta afinidade para o osso e atuam como um 'gancho nos ossos', permitindo direcionamento eficiente $\mathrm{e}$ rápido dos BFs para a superfície do osso. Uma vez localizado dentro do osso, a estrutura e conformação tridimensional da cadeia lateral $\mathrm{R}_{2}$, bem como os grupos fosfonatos, determinam a atividade biológica da molécula e influenciam na capacidade dos fármacos de interagir com alvos específicos ${ }^{12}$

Diversos fármacos da classe dos bifosfonatos foram sintetizados, nos quais os grupos substituintes $R_{1}$ e $R_{2}$ variaram, sistematicamente (Figura 3), com propósitos definidos:

- aumentar a afinidade óssea;

- melhorar o perfil terapêutico: potência, seletividade e toxicidade;

- adquirir nova atividade farmacológica e

- alterar a biodisponibilidade da molécula.

Os BFs que contêm um átomo de nitrogênio primário em uma cadeia alquílica (pamidronato e alendronato) podem ser de 10 a 100 vezes mais potentes que o etidronato e o clodronato, enquanto os derivados destes compostos que contêm um nitrogênio terciário (ibandronato e olpadronato) são, geralmente, mais potentes em inibir a reabsorção óssea ${ }^{16,18-20}$.

Entre os BFs mais potentes que inibem a reabsorção óssea estão aqueles contendo o átomo de nitrogênio em um anel heterocíclico (risedronato e zoledronato) ${ }^{16,18,20}$.

Os primeiros BFs aprovados para uso terapêutico foram clodronato dissódico (doença de Paget), pamidronato dissódico (doença de Paget), alendronato de sódio (osteoporose) e risedronato de sódio (osteoporose). Zoledronato encontra-se em fase experimental para tratamento de câncer de próstata ${ }^{4}$. Além do emprego nessas disfunções, tais compostos podem ser utilizados em hipercalcemia maligna e metástase óssea ${ }^{1,13}$.

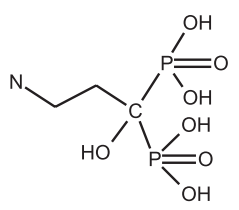

ácido pamidronico

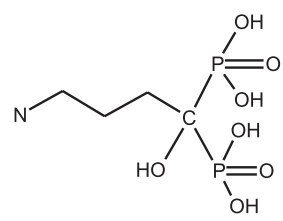

ácido alendronico

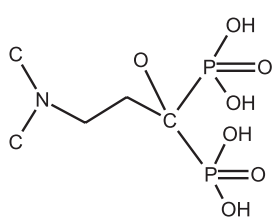

ácido olpadronico

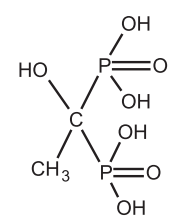

ácido etidronico

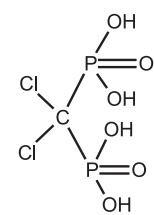

ácido clodronico

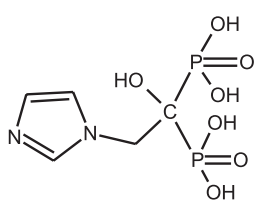

ácido zoledronico
Figura 3. Estruturas representativas da classe de compostos bifosfonatos (BFs)

Recentemente, comprovou-se que os BFs podem agir como substratos de enzimas biosintéticas (por exemplo, proteína tirosina fosfatase, esqualeno sintase, entre outras), sendo incorporados em metabólitos celulares ${ }^{21,22}$.

Em razão de sua propriedade anti-reabsortiva, os BFs também estão sendo investigados como inibidores da destruição das articulações, com possível uso no tratamento da artrite reumatóide (AR). Adicionalmente à sua capacidade de inibir a reabsorção óssea, mostraram atividade antiinflamatória em modelos animais de AR, bem como em humanos ${ }^{23}$.

\section{BIFOSFONATOS COMO TRANSPORTADORES DE RADIOISÓTOPOS}

Radioisótopos dirigidos aos tecidos ósseos têm sido estudados principalmente para fim de diagnóstico. O isótopo radioativo de tecnécio, $99 \mathrm{~m}$-tecnécio ${ }^{99 \mathrm{~m}-\mathrm{Tc}}$ ), foi o isótopo escolhido devido ao seu espectro de emissão satisfatório e meia-vida curta. Os complexos ${ }^{99 m-T c-B F}$ (tecnécio-bifosfonato), formados pela associação física entre ${ }^{99 \mathrm{~m}-\mathrm{Tc}}$ (IV) e um BF, são amplamente usados para imagens de lesões de tumor osteolítico ${ }^{24}$.

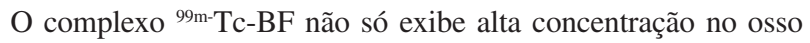
em relação àquela no tecido mole, mas também preferência pela localização na lesão comparativamente àquela no osso normal ${ }^{4}$.

Os BFs inicialmente utilizados como transportadores (Figura 4) foram o 1-hidroxietilideno-1,1-difosfonato (HEDP), metileno-1,1difosfonato (MDP), 1-hidroximetano-1,1-difosfonato (HMDP) e 2,3dicarboxipropano-1,1-difosfonato ${ }^{4,25}$.

Além do emprego em diagnóstico, os complexos BFs-radioisótopos direcionados aos ossos têm sido crescentemente explorados para fins terapêuticos em pacientes com tumor ósseo. Os resultados terapêuticos foram alívio da dor e inibição da progressão do tumor $^{26}$.

Outros radioisótopos utilizados neste sistema são ${ }^{131} \mathrm{I}$ e ${ }^{211} \mathrm{At}$, 


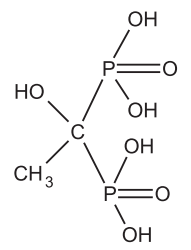

HEDP

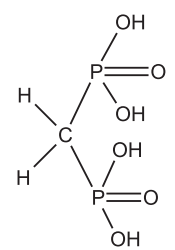

MDP

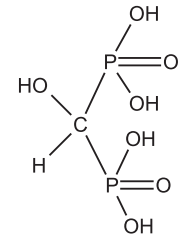

HMDP
Figura 4. Estruturas químicas de transportadores bifosfonatos

devido à alta estabilidade da ligação isótopo-BF, permitindo melhor eficácia de direcionamento e retenção osteolítica local ${ }^{27}$. Os BFs utilizados em complexos com esses isótopos são mostrados na Figura 5.
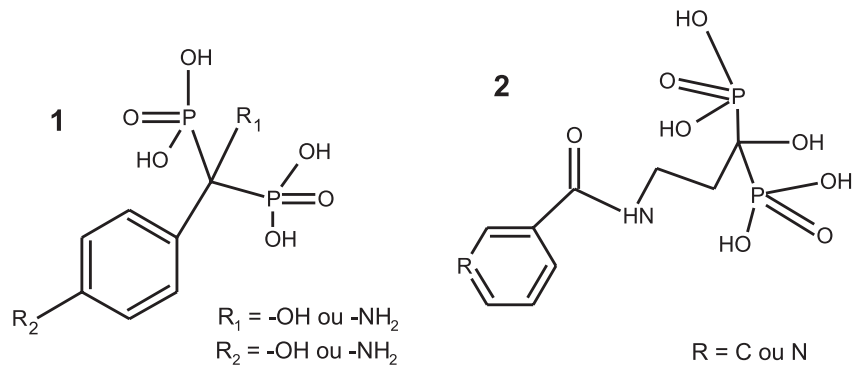

Figura 5. Os BFs 1 e 2 são usados para a liberação de radioisótopos ${ }^{131} I$ e ${ }^{211}$ At, que estão ligados ao anel benzênico

\section{AGENTES ANTINEOPLÁSICOS DIRIGIDOS PARA OS OSSOS}

Os BFs previnem a atividade osteolítica induzida por tumor, mas são, geralmente, ineficazes como agentes antineoplásicos contra metástase óssea. Os agentes antineoplásicos, por outro lado, apresentam ação limitada na metástase óssea, devido ao extenso volume de distribuição resultante de efeitos secundários em locais extraesqueleto. Um complexo BF-agente antineoplásico tem ação potencial como um fármaco para câncer de tumores localizados em tecidos ósseos. Tais tumores podem-se originar em ossos ou podem surgir como um resultado da expansão metastásica de outros órgãos, como seio e próstata ${ }^{28}$.

A Figura 6 mostra a estrutura de alguns pró-fármacos antineoplásicos.

A pesquisa inicial sobre BFs citotóxicos foi relatada, em 1986, por Winger e colaboradores ${ }^{29}$. O primeiro conjugado, diglicidil-[3(3,3-difosfonato-3-hidroxipropilamino)-2-hidroxipropil]-urazol (DDU), consistiu na combinação de pamidronato e 1,2,4-triglicidilurazol, atingindo-se direcionamento ósseo similar ao do pamidronato $^{30}$.

Um segundo conjugado, 4-[4-[bis(2-cloroetil)amino]fenil]-1hidroxibutano-1,1-ácido bifosfônico (BAD), foi preparado pela derivatização do melfalano com um $\mathrm{BF}^{31}$. Embora o BAD apresente atividade, a eficácia não foi claramente superior à do melfalano, uma vez que altas doses do composto foram necessárias para se observar o efeito terapêutico.

A versão modificada de BAD, 3-[bis-(2-cloroetil)-amino]-4metilfenil-hidroximetano-1,1-ácido bifosfônico (BCMP) foi também preparada, mostrando maior efeito citostático do que aquele apresentado pelo $\mathrm{BAD}^{32}$.

Análogos de cisplatina, como o AMDP, foram preparados usando modelo de osteossarcoma transplantado. Este foi tão eficaz quanto a cisplatina na redução do volume do tumor e mais ativo que a<smiles>CC(CNCCC(N)(O)CO)CN1CN(CC2CO2)N(CC2CO2)C1</smiles><smiles>Cc1ccc(C(O)([18OH])[18OH])cc1N(CCCl)CCCl</smiles>

BCMP

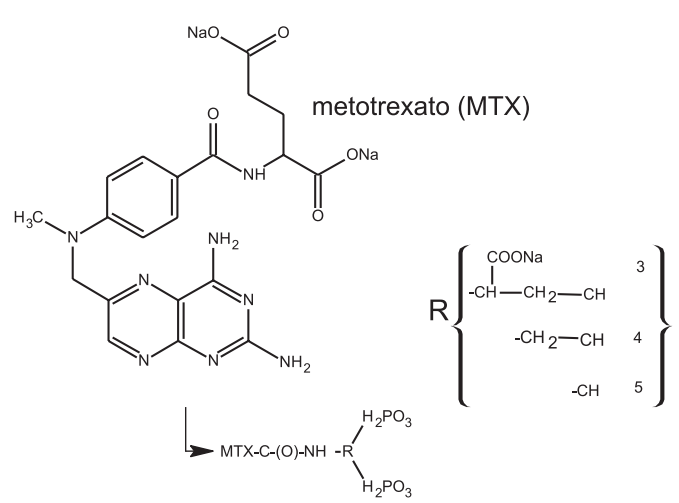

Figura 6. Agentes antineoplásicos conjugados a BFs cisplatina no prolongamento da vida. Também, mostrou-se mais eficaz que pamidronato, 3- $N, N$-dimetilamino-1-hidroxipropilideno-1,1difosfonato e 3-N,N-dimetilamino-1,1-hidroxibutilideno-1,1difosfonato, retardando o crescimento do tumor em modelo animal ${ }^{33}$.

Conjugados metotrexato-BF (Figura 6) foram estudados por Sturtz e colaboradores ${ }^{34}$, que observaram que os conjugados 3 e 4 apresentavam atividade superior em doses menores comparativamente aos derivados 4-5.

\section{LIBERAÇÃO DE AGENTES TERAPÊUTICOS PARA AUMENTAR A MASSA ÓSSEA}

Agentes terapêuticos capazes de inibir a reabsorção e estimular a formação óssea são duas classes de substâncias cuja eficácia pode ser significativamente aumentada através da liberação seletiva para os ossos. São benéficos em situações clínicas nas quais o aumento do tecido mineralizado é necessário devido ao desequilíbrio metabólico sistêmico, como na osteoporose pós-menopausa. Eles podem, também, ser úteis em condições degenerativas locais, como na doença de Paget, osteólise de tumor local ou osteonecrose, em que a regeneração óssea local necessita de estímulo ${ }^{4}$.

Três conjugados de $17 \beta$-estradiol e alendronato, com ligações sensíveis a esterases, foram preparados por Bauss e colaboradores ${ }^{34}$, como agentes osteotrópicos. Os conjugados diferem no tipo de ligação entre o $17 \beta$-estradiol e o alendronato, levando a uma diferença na velocidade de cisão do pró-fármaco para formação da porção ativa, no soro ${ }^{4,34}$.

A cisão da ligação éster dos conjugados de $17 \beta$-estradiol-BF encontra-se dependente da porção adjacente da ligação éster. O derivado do ácido dimetilglutárico (6, Figura 7) não foi metabolizado, ao contrário dos derivados do ácido dimetilsuccínico e cicloexano 
(7 e 8, respectivamente, Figura 7), que ocorreram na extensão de 4,8 e $33 \%$, respectivamente, em 48 h. Estes dados estão de acordo com os resultados de atividade biológica no estímulo da formação óssea, sendo o composto 6 inativo. Este estudo sugere a necessidade de metabolização lenta da ligação éster para que haja a liberação do $17 \beta$-estradiol ${ }^{34}$.

Outros dois BFs conjugados com 17 $\beta$-estradiol, 9 e $\mathbf{1 0}$ (Figura 7), foram também relatados por Page et al. ${ }^{35,36}$, onde a porção bifosfônica foi colocada nas posições 3 ou 17 deste fármaco, porém, seu efeito biológico não foi demonstrado.

Além de $17 \beta$-estradiol, Gil et $a l .{ }^{37}$ relataram o estudo de uma série de conjugados $\mathrm{BFs}$ e prostaglandina $\mathrm{E}_{2}\left(\mathrm{PGE}_{2}\right)$, planejados para estimular a formação óssea na osteoporose. $\mathrm{A} \mathrm{PGE}_{2}$ é um agente anabólico, que tem sido usado em combinação com BFs (alendronato e risedronato) para estimular a formação óssea ${ }^{38,39}$.

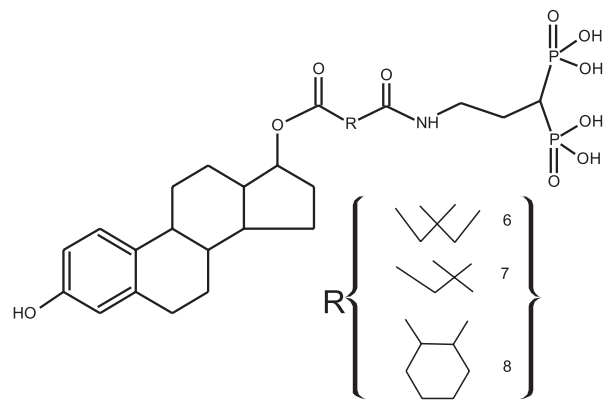

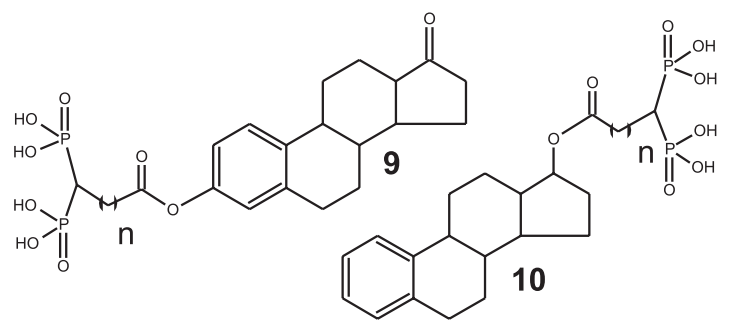

Figura 7. Conjugados de 17- $\beta$-estradiol ineficaz (6) e eficazes (7-10) para a prevenção da perda óssea

\section{AGENTES ANTIINFLAMATÓRIOS DIRIGIDOS PARA OS OSSOS}

O primeiro relato de um fármaco antiinflamatório não-esteróide (AINE) planejado para ser direcionado aos ossos foi em 1991. Consistiu na conversão do grupo carboxila do pirazolaco em uma porção bifosfônica. O conjugado mostrou-se eficaz em modelos de ratos e cavalos artríticos. Entretanto, diferenças na via e dose administrada (oral vs. intravenosa) não permitiram comparação da eficácia do pirazolaco e do derivado com o $\mathrm{BF}^{40}$.

Conjugados de BFs com corticosteróides, como prednisolona, hidrocortisona e cortodoxona, também foram preparados por Guervenou e Strurtz ${ }^{41}$, em 1994 (11, Figura 8).

O diclofenaco (DIC) é usado clinicamente no tratamento do reumatismo articular crônico mas, para se obter bons resultados terapêuticos, são inevitáveis administrações repetidas. Como o uso desse, assim como de outros AINEs, leva a graves danos gastrintestinais, Hirabayashi e colaboradores ${ }^{1}$, sintetizaram um novo prófármaco bifosfônico do DIC (DIC-BF - Figura 9), demonstrando as propriedades de tropismo ósseo e liberação prolongada do prófármaco DIC-BF (Figura 10) que, além de aumentar os efeitos farmacológicos do diclofenaco, preveniu, simultaneamente, os efeitos tóxicos gastrintestinais. Esses pesquisadores também concluí-

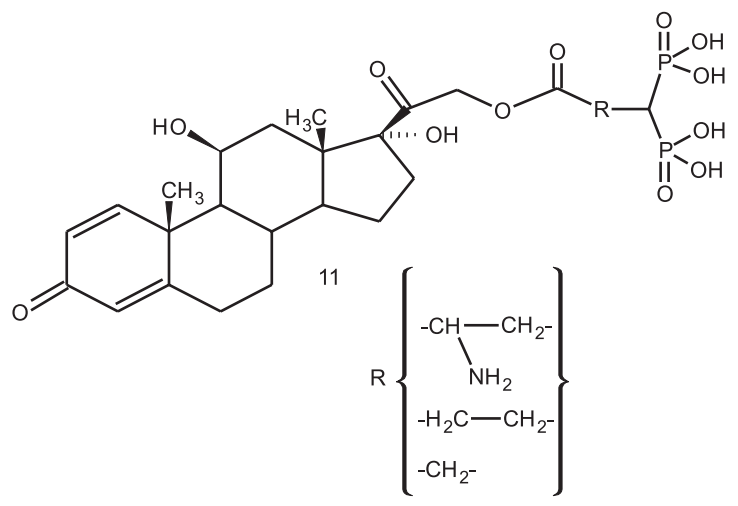

Figura 8. Prednisona (antiinflamatório esteróide) latenciado com bifosfonato

ram que o sistema ODDS do diclofenaco era promissor como método para se alcançar alta eficácia, reduzindo o número de administrações.<smiles>CCOP(=O)(O)C(NC(=O)COC(=O)Cc1ccccc1Nc1c(Cl)cccc1Cl)P(=O)(O)OCC</smiles>

Figura 9. Pró-fármaco diclofenaco-bifosfonato (DIC-BF)

\section{PROTEÍNAS DIRIGIDAS AOS OSSOS}

Agentes terapêuticos baseados em proteínas e peptídios são utilizados para aumentar a aceitação do tratamento de doenças musculoesqueléticas. A calcitonina, hormônio da paratireóide, e agentes inflamatórios de inibição protéica (Fator de Necrose Tumoral e Interleucina-1) são efetivos no tratamento de osteoporose e artrite reumatóide, respectivamente. Entretanto, o uso clínico tem sido limitado por duas barreiras fundamentais: meia-vida pequena na circulação devido à sua rápida remoção para o fígado, rins e baço, e efeitos colaterais indesejáveis ${ }^{30,42}$.

A idéia de BFs conjugados a proteínas foi divulgada através de uma patente utilizando polietilenoglicol (PEG) hidrofílico como ligante. Entretanto, a afinidade aos ossos não foi comprovada ${ }^{43}$.

Uludag e colaboradores ${ }^{44}$, utilizando albumina sérica bovina (ASB) como proteína modelo, demonstraram in vitro que a afinidade da proteína modificada é diretamente proporcional à extensão de conjugação (5 BFs por proteína). O conjugado aminoBF (BF-ASB) mostrou ter alta afinidade óssea in vivo após injeção intra-óssea (tíbia) em ratos $^{30}$.

\section{CONCLUSÃO}

Pode-se concluir que a utilização do sistema bifosfonato como transportador para os ossos (ODDS), apesar de recente e ainda em experimentação, apresenta grandes vantagens devido às características do próprio osso e sua localização, que dificultam a ação dos fármacos. Constitui-se em sistema, com certeza, muito promissor de transporte de fármacos, digno de estudos que envolvam o direcionamento de compostos e, conseqüentemente, a seletividade destas 

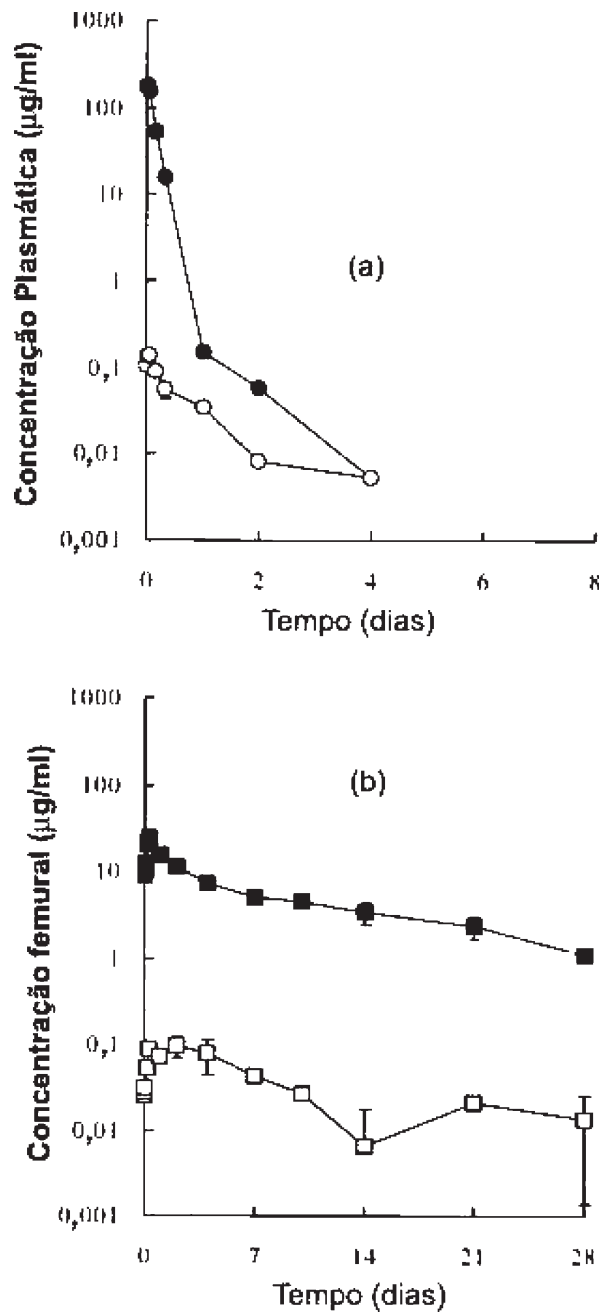

Figura 10. Tempo de concentração no plasma (a) e fêmur (b) do DIC-BP e regeneração de DIC 28 dias após injeção intravenosa de DIC-BP em ratos com uma dose de $10 \mathrm{mg} / \mathrm{kg}$. Os símbolos cheios e vazios representam DICBP e DIC regenerado, respectivamente. Reproduzida da ref. 1, com permissão da Elsevier

substâncias nos ossos, aumentando, significativamente, a esperança de se encontrar derivados eficazes e menos tóxicos com aplicação terapêutica em doenças ósseas.

\section{REFERÊNCIAS}

1. Hirabayashi, H.; Takahashi, T.; Fujisaki, J.; Masunaga, T.; Sato, S.; Hiroi, J.; Tokunaga, Y.; Kimura, S.; Hata, T.; J. Controlled Release 2001, 70, 183.

2. Franceschi, R. T.; Crit. Rev. Oral Biol. Med. 1999, 10, 40.

3. Lian, J. B.; Stein, G. S.; Canalis, E.; Robey, P.; Boskey, G. A. Em Primer on the Metabolic Bone Diseases and Disorders of Bone Metabolis; $4^{\text {th }}$ ed.; Favus, M. J., ed.; Lippincott Williams \& Williams: Philadelphia, 1999.

4. Uludag, H.; Curr. Pharm. Des. 2002, 8, 99.

5. Ducy, P.; Schinke, T.; Karsenty, G.; Science 2000, 289, 1501.

6. Teitelbaum, S. L.; Science 2000, 289, 1504.

7. Coleman, R. E.; Cancer Treat. Rev. 2001, 27, 165.
8. Bauman, W. A.; Spungen, A. M.; Phys. Med. Rehabil. Clin. N. Am. 2000, 11,109 .

9. Rehman, Q.; Lane, N. E.; Arthritis Res. 2001, 3, 221.

10. Fujisaki, J.; Tokunaga, Y.; Takahashi, T.; Hirose, T.; Shimojo, F.; Kagayama, A.; Hata, T.; J. Drug Targeting 1995, 3, 273.

11. Fleisch, H.; Russell, R. G. G.; Straumann, F.; Nature 1966, 212, 901.

12. Rogers, M. J.; Frith, J. C.; Luckman, S. P.; Coxon, F. P.; Benford, H. L.; Monkkonen, J.; Auriola, S.; Chilton, K. M.; Russell, G. G.; Bone 1999, $24,73 \mathrm{~S}$

13. Ezra, A.; Golomb, G.; Adv. Drug Delivery Rev. 2000, 42, 175.

14. Ebranhimpour, A.; Francis, M.D. Em Bisphosphonate Therapy in Acute and Chronic Bone Loss: Physical Chemical Considerations in Bisphosphonate-related Therapies; Bijvoet, O.; Fleisch, H. A.; Canfield, R. E.; Russell, R. G. G., eds.; Bisphosphonate on Bones, Elsevier: Amsterdam, 1995.

15. Russell, R. G. G.; Muhlbauer, R. C.; Bisaz, S.; Williams, D. A.; Fleisch, H.; Calcif. Tissue Res. 1970, 6, 183.

16. Russell, R. G. G.; Rogers, M. J.; Bone 1999, 25, 97.

17. van Beek, E.; Hoekstra, M.; van der Ruit, M.; Löwik, C.; Papapoulos, S.; J. Bone Miner. Res. 1994, 9, 1875.

18. Geedes, A. D.; D'souza, S. M.; Ebetino, F. H.; Ibbotson, K. J.; J. Bone Miner. Res. 1994, 8, 265.

19. Shinoda, H.; Adamek, G.; Felix, R.; Fleisch, H.; Schenk, R.; Hagan, P.; Calcif. Tissue Int. 1983, 35, 87.

20. Schenk, R.; Eggli, P.; Fleisch, H.; Rosini, S.; Calcif. Tissue Int. 1986, 38, 342 .

21. Russell, R. G.; Rogers, M. J.; Frith, J. C; Luckman, S. P.; Coxon, F. P.; Benford, H. L.; Croucher, P. I.; Shipman, C.; Fleisch, H. A.; J. Bone Miner. Res. 1999, 14, 53.

22. Rogers, M. J.; Gordon, S.; Benford, H. L.; Coxon, F. P.; Luckman, S. P.; Monkkonen, J.; Frith, J. C.; Cancer 2000, 88, 2961.

23. Makkonen, N.; Salminen, A.; Rogers, M. J.; Frith, J. C.; Urtti, A.; Azhayeva, E.; Mönkkönen, J.; Eur. J. Pharm. Sci. 1999, 8, 109.

24. De Ligny, C. L.; Gelsema, W. J.; Tiji, T. G.; Huigen, Y. M.; Vink, H. A.; Nucl. Med. Biol. 1990, 17, 161.

25. Atkins, H. L.; Srivastava, S. C.; Q. J. Nucl. Med. 1996, 40, 285.

26. McEwan, A. J.; Sem. Rad. Oncol. 2000, 10, 103.

27. Eisenhut, M.; J. Nucl. Med. 1984, 25, 1356.

28. Coleman, R. E.; Breast Cancer 2000, 7, 361

29. Winger, F.; Eichmann, T.; Manegold, C.; Krempien, B.; J. Cancer Res. Clin. Oncol. 1986, 111, 35

30. Winger, F.; Sterz, H.; Blum, H.; Moller, H.; Pittermann, W.; Pool, B. L.; Sinn, H. J.; Spring, H.; Schmahl, D.; J. Cancer Res. Clin. Oncol. 1986, $111,209$.

31. Pool, B. L.; Berger, M.; Schlehofer, J. R.; Winger, F.; Invest. New Drugs 1988, 6, 67.

32. Klenner, T.; Winger, F.; Keppler, B. K.; Krempien, B.; Schmahl, D.; J. Cancer Res. Clin. Oncol. 1990, 116, 341.

33. Sturtz, G.; Appere, G.; Breistol, K.; Fodstad, O.; Schwartsmann, G.; Hendriks, H. R.; Eur. J. Med. Chem. 1992, 27, 825.

34. Bauss, F.; Esswein, A.; Reiff, K.; Sponer, G.; Muller-Beckmann, B.; Calcif. Tissue Int. 1996, 59, 168.

35. Page, P. C. B.; Mckenzie, M. J.; Gallagher, J. A.; J. Org. Chem. 2001, 66, 3704.

36. Page, P. C. B.; Moore, J. P. G.; Manfield, I.; Mckenzie, M. J.; Bowler, W. B. J. A.; Tetrahedron 2001, 57,1837.

37. Gil, L.; Han, Y.; Opas, E. E.; Rodan, G. A.; Ruel, R.; Seedor, J. G.; Tyler, P. C.; Young, R. N.; Bioorg. Med. Chem. 1999, 7, 901.

38. Humpel, M.; Gunzel, P.; Biere, H.; Junginger, B.; Agents Actions 1991, $32,22$.

39. Lin, B. Y.; Jee, W. S.; Ma, Y. F.; Ke, H. Z.; Kimmel, D. B.; Li, X.; Bone 1994, 15, 489 .

40. Guervenou, J.; Strurtz, G.; Phosp. Sulp. Sil. 1994, 8, 1.

41. Gittens, S.; Uludag, H.; J. Drug Target 2001, 6, 10.

42. Bentz, H.; Rosen, D.; EP 0512 844A1 1992.

43. Uludag, H.; Kousinioris, N.; Gao, T., Kantoci, D.; Biotechnol. Prog. 2000, $16,258$.

44. Uludag, H.; Yang, J.; Biotechnol. Prog. 2002, 18, 604. 